\title{
The Galaxy Merger Origin of Hot Gaseous Halos of Ellipticals
}

\author{
X.Y. Xia \\ Dept. of Physics, Tianjin Normal University, Tianjin, 300074, PRC
}

Z.Y. Huo, S.J. Xue

National Astronomical Observatories, Chinese Academy of Science, A20 Datun Road, 100012, Beijing, PRC

\begin{abstract}
.
We report on the properties of the hot gaseous halos of 10 nearby ultraluminous IRAS galaxies from Chandra observations. There exists diffuse soft X-ray emission surrounding the central nucleus within $10 \mathrm{kpc}$ of the nuclear region with a temperature of about $0.7 \mathrm{keV}$ and metallicity about $Z \sim 1.0 Z_{\odot}$. Also, emission lines from $\alpha$ elements and ions for are seen in all nearby ULIRGs in our sample. Outside the central region, the Chandra observations reveal a more extended hot gaseous halo with a temperature of about $0.6 \mathrm{keV}$ and low metallicity $\left(Z \sim 0.1 Z_{\odot}\right)$ for some of the ULIRGs. We discuss the nature and the implications of our results for the origin of X-ray halos in elliptical galaxies and the constraints this places on the feedback process.
\end{abstract}

\section{Introduction}

It is widely accepted that Ultraluminous Infrared Galaxies (hereafter ULIRGs, see Sanders \& Mirabel 1996, for a review) belong to a population of objects at an important evolutionary stage going from strong galaxy interactions/mergers to the formation of elliptical galaxies through massive starburst and AGN phases. Although the nature of ULIRGs is believed to be understood in general terms, it is still not clear whether all these objects originate from interactions/mergers of a pair of gas-rich spirals or even galaxy groups, and therefore, whether some ULIRGs evolve to become giant elliptical galaxies through a bright QSO phase with galaxy groups as their progenitor.

There is mounting observational evidence to support the scenario of elliptical galaxy formation by multiple merging from the identification of the number of nuclei of merging galaxies (e.g., Borne 2000; Cui et al. 2001, and references therein). X-ray observations can provide an independent way to distinguish the progenitor of mergers because if the progenitor of a merger is a group of galaxies, the X-ray halo of the group must be preserved quite a long time after merging. The fossil groups with luminous spatially extended X-ray emission are supposed to be the end result of merging within a normal group (Jones et al. 2003). If the extended diffuse X-ray emission of fossil groups and some ULIRGs are similar, 

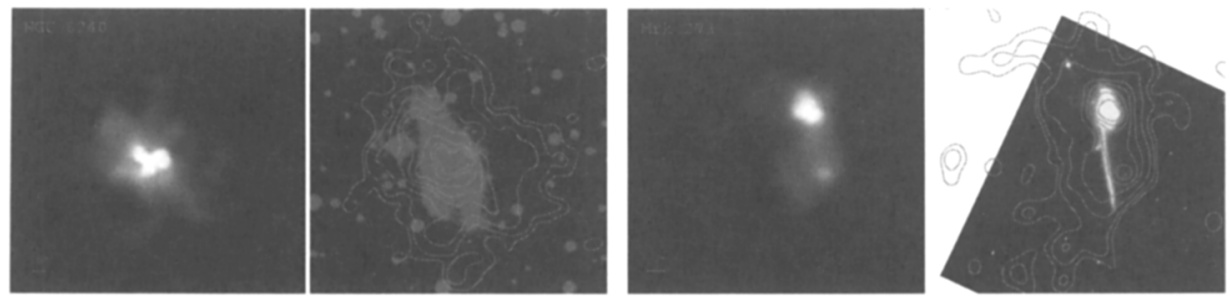

Figure 1. The soft X-ray images (left panels) and optical images overlaid with soft X-ray contours (right panel).

there might exist some evolutionary connection among compact groups, ULIRGs and fossil groups.

On the other hand, elliptical galaxies are known to have hot gas halos; however, the properties of hot gas halos are very diverse. X-ray luminous galaxies show very extended emission and the X-ray luminosity of X-ray bright ellipticals shows large scatter. It is now clear, based on Chandra observations, that the soft X-ray emission of early type galaxies detected by ROSAT is mainly due to diffuse hot gas. Recently, O'Sullivan et al. (2003) suggested, based on an amalysis of 39 X-ray luminous early type galaxies, that the X-ray halo of elliptical galaxies is not formed by infall and shock heating of primordial gas. Instead, it seems likely that the hot gas halo of ellipticals is produced by stellar mass loss within galaxies. However, from the results of O'Sullivan et al. (2003), the metallicities of luminous early type galaxies cover quite a large range. It is unreasonable that very low metallicity hot gas is from stellar mass loss. As elliptical galaxies formed by galaxy mergers, foloowing the hierarchical galaxy formation scenario, the significantly different properties in their hot gas halo might imply that the origin of the hot gas of early type galaxies and the progenitors of elliptical galaxies are different.

Chandra and XMM-Newton have observed more than 15 luminous or ultraluminous IRAS galaxies (Ptak et al. 2003; Franceschini et al. 2003). Investigating the hot gas halo properties for an ULIRGs sample at different merging states based on sub-arcsecond resolution using the Chandra database should provide clues to an understanding of the origin of hot gas halos of elliptical galaxies as well as the metal enrichment and energy ejection processes.

Throughout this paper, we use a Hubble constant of $H_{0}=65 \mathrm{~km} \mathrm{~s}^{-1} \mathrm{Mpc}^{-1}$, and an $\Omega_{m}=0.3$ and $\Omega_{\lambda}=0.7$ cosmology.

\section{Sample and Data Reduction}

The purpose of this work is to study the diffuse hot gaseous halo properties. We need spatially resolved datasets and enough detected photons for analyzing diffuse hot gas. Therefore, we use Chandra archive data on the ULIRGs sample used by Franceschini et al. (2003) plus Arp 299; all sample ULIRGs have redshifts of about or less than 0.04 . In total the sample includes 10 ULIRGs, of which 4 have a single nucleus and 6 have double nuclei. 

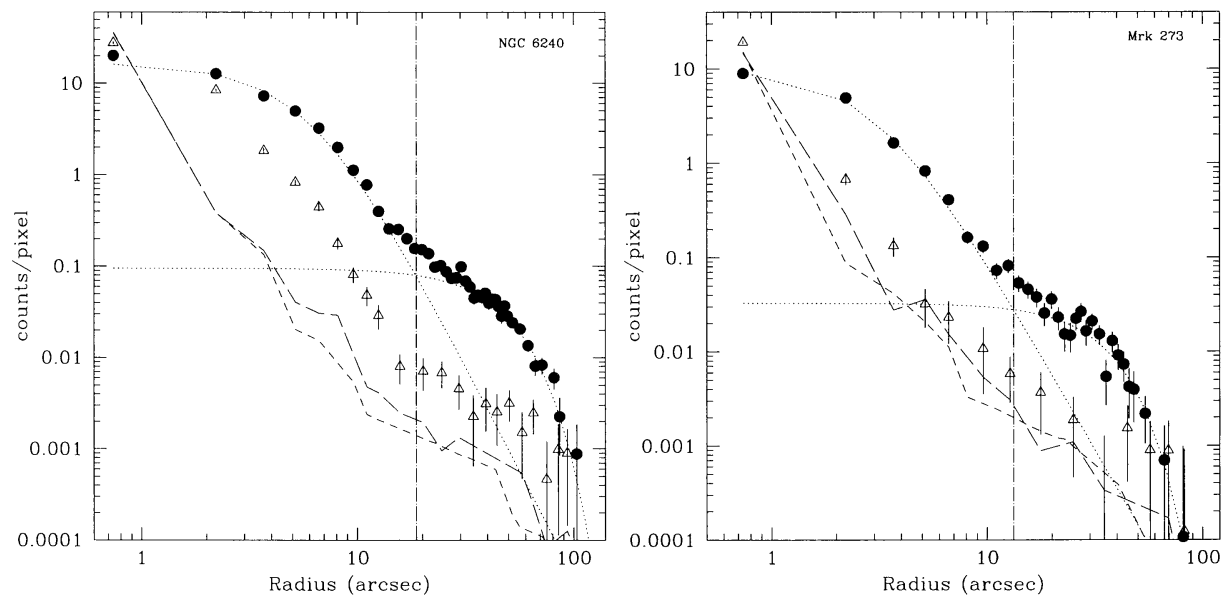

Figure 2. The surface brightness profiles for NGC 6240 and Mrk 273; the soft and hard X-ray points are shown as filled circles and triangles, respectively.

Generally, the data reduction was performed using CIAO 2.3 and the data were reprocessed using CALDB 2.21 from the level 1 event file. We also apply the CTI correction when the temperature of the Focal Plane at the time of the observation was $153 \mathrm{~K}$. Sources are detected using wavdetect, the background light curve is examined and the background flaring is removed. The data are filtered to include only the standard event grades $0,2,3,4,6$.

$\mathrm{X}$-ray emission is detected for all sources. The data were analyzed in full (0.3-8.0 keV), soft (0.5-2.0 keV) and hard (2.0-8.0 keV) bandpasses, respectively.

\section{Image and Surface Brightness Profiles}

X-ray images of ULIRGs at both soft (0.3-2 keV) and hard X-ray (2-8 keV) bands are adaptively smoothed using the CIAO task csmooth at the $3 \sigma$ significance level and scaled logarithmically. It is obvious from all images that the soft $\mathrm{X}$-ray emission encompasses the main optical images, even entire structures including the long tidal tails for some sample ULIRGs. Therefore the soft X-ray emission for these ULIRGs is clearly extended. The most striking feature from the X-ray images of ULIRGs is that for most objects the central diffuse emission is significantly brighter than the outer diffuse emission after subtracting point sources (central AGN or other X-ray point sources).

From the background subtracted soft $(0.3-2 \mathrm{keV})$ and hard $(2-8 \mathrm{keV})$ X-ray images, we extract the azimuthally-averaged radial surface brightness profiles for all sample ULIRGs using the CIAO task dmextract.

Fig. 1 shows the soft X-ray images and Fig. 2 shows the surface brightness profiles as well as the PSFs for both soft and hard X-ray bands for NGC 6240 and Mrk 273. We can see clearly from Fig. 1 and Fig. 2 that these two ULIRGs have very extended diffuse soft X-ray emission. Actually, all sample ULIRGs 

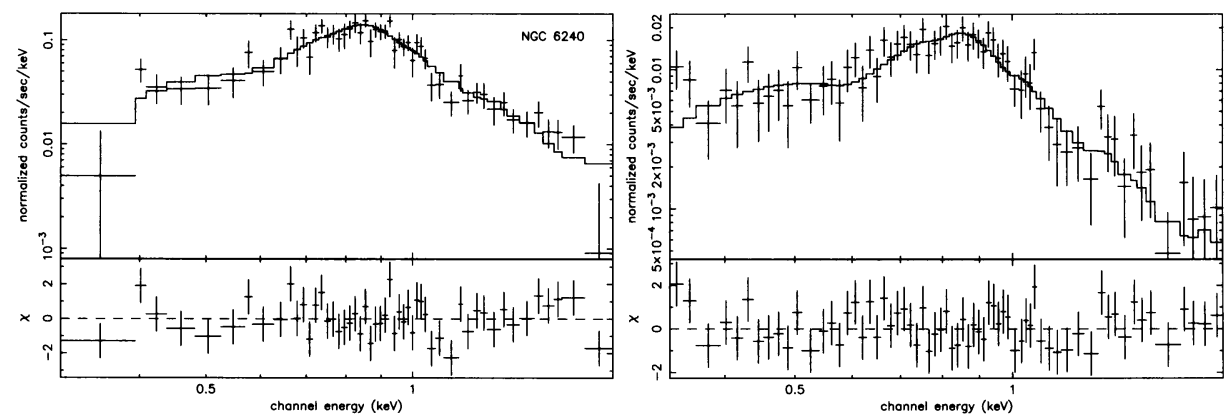

Figure 3. The spectra of the "big halo" for NGC 6240 (left panel) and the stacked data of Mrk 273, Mrk 231 and Arp 220 (right panel).

have diffuse soft X-ray emission. Moreover, the extended hard X-ray emission on scales of about $10 \mathrm{kpc}$ is also detected for some objects, as has been discussed by Franceschini et al. (2003). Furthermore, the break in the surface brightness profiles is common for the sample ULIRGs, although it is more significant for those ULIRGs with a very extended hot gas halo. We use a $\beta$ model to fit the surface brightness profiles. Two $\beta$ models can fit the surface brightness profiles well for Mrk 273, Mrk 231, NGC 6240 and Arp 220. One $\beta$ model can fit the stacked surface brightness profile of the remaining 5 ULIRGs. The break points in the surface brightness profiles are pointed out by vertical lines in Fig. 2. The break may indicate the different properties of two separated diffuse hot gaseous halos. For convenience, we call the inner bright soft X-ray diffuse gas the "small halo" and the outer more extended soft X-ray diffuse gas the "big halo" in the following sections. The physical scales of the "small halo" are about $10 \mathrm{kpc}$ and those of the "big halo" are as large as a few tens of kpc for some ULIRGs.

\section{Spectral Analysis}

Given the sub-arcsecond resolution of Chandra, we can perform spatially-resolved spectral analysis on the diffuse hot gaseous halos for ULIRGs. We extract the 0.3-2 keV spectra for the "big halo" and 0.3-8 keV spectra for the "small halo" as emission within the $2-8 \mathrm{keV}$ range only extends to the region of the "small halo" for ULIRGs with extended hard X-ray emission. Then we performed spectral analysis with the XSPEC (V11.20) and FTOOLS 5.2 software.

\subsection{The "Big Halo"}

Chandra has not detected the soft X-ray "big halo" for IRAS 05189-2524; the spectral analysis for the "big halo" is only done for 9 ULIRGs. The MEKAL thermal model gives acceptable fits to the spectra for 7 target ULIRGs.

The fitting results show that the temperatures of the "big halo" are about $0.6 \mathrm{keV}$ and the metallicity is about 0.1 solar for NGC 6240, Mrk 273, Mrk 231, Arp 220 and Arp 299, which confirms the result we got based on Chandra observations for Mrk 273 (Xia et al. 2002). In order to get more convincing 
results for the "big halo", we performed a stacking analysis on Mrk 273, Mrk 231 and Arp 220; NGC 6240 has a high enough number of photons detected (more than 2500 photons detected within the "big halo"). Arp 299 was observed by the ACIS-I instead of the ACIS-S detector and the number of photons detected for the "big halo" of IRAS 17218-0014 and IRAS 20551-4250 is too low to play a role in the stacking analysis. It is remarkable from Fig. 3 that the spectrum of NGC 6240 and the stacked spectrum are very similar; the fitting results indicate that the temperature and metallicity of the "big halo" for NGC 6240 and the stacked data of Mrk 273, Mrk 231, Arp 220 (with more than 1200 photons) are almost the same, namely with a temperature of $0.6 \mathrm{keV}$ and metallicity of 0.1 solar.

\subsection{The "Small Halo"}

As the soft X-ray emission within the "small halo" is from diffuse hot gas, X-ray binaries and AGN, the spectral analysis of the "small halo" is much more complicated. The fitting models for all sample ULIRGs are more or less consistent with published results. Given that our main interest in this paper is to study the properties and the origin of diffuse hot gas instead of the AGNs, we only pay attention to the spectral fitting parameters with MEKAL for a thermal plasma emission component. The most intriguing result, compared with the "big halo" is that the metallicities of the "small halo" for all sample ULIRGs are about equal to or higher than the solar value which is completely different for the metallicity of the "big halo".

\section{Discussion and Summary}

Given the completely different metallicities for the "small halo" and the "big halo" of ULIRGs, there is no doubt that the two components of diffuse hot gaseous halos have different origins, namely, the "small halo" with high metallicity has a stellar origin and is enriched by a superwind during a massive starburst, while the low metallicity "big halo" is from secondary infall of primordial gas.

Fig. 4 shows the Lx-T relation for both the "big halo" (left panel) and "small halo" (right panel) of ULIRGs. For comparison, we also plot elliptical galaxies of O'Sullivan et al. (2003) and groups of galaxies of Xue \& Wu (2000). From Fig. 4, both the "big halo" and "small halo" of the hot diffuse gas detected in ULIRGs coincides very well with the emission of elliptical galaxies or groups of galaxies, which implies a possible evolutionary connection among groups of galaxies, mergers and bright elliptical galaxies.

The energy feed back to the ISM and metal enrichment process by superwinds is another largely unconstrained problem concerning galaxy formation. Investigating such processes happening in massive starburst galaxies by Chandra is therefore essential. The radius of the inner high metallicity hot gas halos for all sample ULIRGs is about $10 \mathrm{kpc}$ or less, regardless if it is in the process of merging or has merged. On the other hand, the outer extended hot gaseous halos for all ULIRGs have very low metallicity. This indicates that the ISM of the outer part of the merger is less metal-enriched and that the metals produced and ejected in the central star formation regions are just mixed with the inter- 

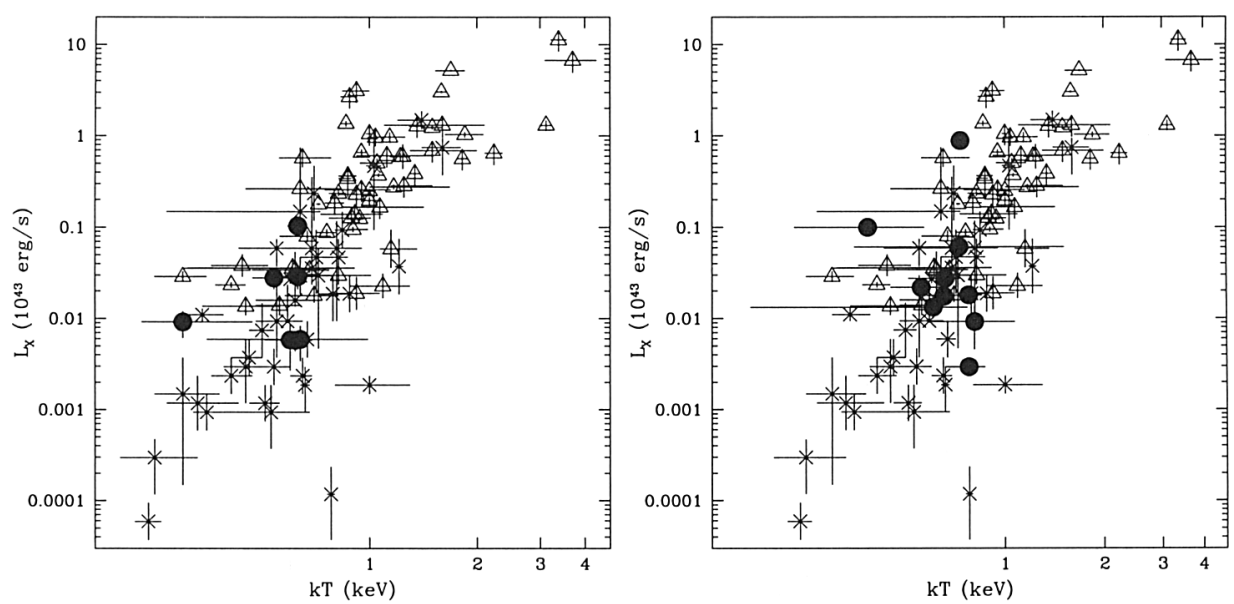

Figure 4. The Lx-T relation for a sample of ULIRGs (filled circles), groups of galaxies (triangles) and ellipticals (crosses); Left panel: "big halo"; Right panel: "small halo".

stellar medium within a $10 \mathrm{kpc}$ region after the system has almost finished its merger. As ULIRGs are massive starburst galaxies with strong superwinds, this fact sets constraints on the efficiency of the feedback process.

Acknowledgments. We are grateful to the CXC team for help with the data reduction. We also thank Profs. Z.G. Deng, S. Mao, X.P. Wu and J. Hibbard for advice and helpful discussions. This project was supported by the NSF of China and TG1999075404.

\section{References}

Borne, K.D., Bushouse, H., Lucas, R.A., \& Colina, L. 2000, ApJ, 529, L77

Cui, J., Xia, X.-Y., Deng, Z.-G., Mao, S., Zou, Z.L. 2001, AJ, 122, 63

Jones, L.R., Ponman, T.J., Horton, A., Babul, A., Ebeling, H. \& Burke, D.J. 2003, MNRAS, 343, 627

Franceschini, A. et al. 2003, MNRAS, 343, 1181

Strickland, D.K., Heckman, T. M., Colbert, E.J.M., Hoopes, C.G., \& Weaver, K.A. 2003, astro-ph/0306592

O'Sullivan, E., Ponman, T. J. \& Collins, R.S. 2003, MNRAS, 340, 1375

Ptak, A., Heckman, T., Levenson, N. A., Weaver, K., Strickland, D. 2003, ApJ, 592,782

Sanders, D.B., \& Mirabel, I.F. 1996, ARA\&A, 34, 749

Xia, X. Y., Xue, S. J., Mao, S., Boller, Th., Deng, Z. G., \& Wu, H. 2002, ApJ, 576,738

Xue, Y. J., \& Wu, X. P. 2000, ApJ, 538, 65 